\title{
Effect of interleukin 6 deficiency on the expression of Bcl-2 and Bax in the murine heart
}

\author{
Karol A. Kamiński ${ }^{1}$, Marcin Kożuch ${ }^{2}$, Tomasz A. Bonda ${ }^{1,3}$, \\ Maria M. Stepaniuk ${ }^{1,4}$, Ewa Waszkiewicz ${ }^{1}$, Lech Chyczewski ${ }^{4}$, \\ Włodzimierz J. Musiał' ${ }^{1}$, Maria M. Winnicka ${ }^{3}$ \\ ${ }^{1}$ Department of Cardiology, Medical University of Białystok, M. Curie-Skłodowskiej 24A, PL 15-276 Białystok, Poland \\ ${ }^{2}$ Department of Invasive Cardiology, Medical University of Białystok, M. Curie-Skłodowskiej 24A, \\ PL 15-276 Białystok, Poland \\ ${ }^{3}$ Department of General and Experimental Pathology, Medical University of Białystok, A. Mickiewicza 2C, \\ PL 15-222 Białystok, Poland \\ ${ }^{4}$ Department of Clinical Molecular Biology, Medical University of Białystok, Waszyngtona 13, PL 15-276 Białystok, \\ Poland
}

Correspondence: Maria M. Winnicka, e-mail: mmw@amb.edu.pl

\begin{abstract}
:
Interleukin 6 (IL-6) is a pleiotropic cytokine that is highly expressed in response to ischemia and reperfusion. It has dichotomous roles in the heart, functioning both as an inflammatory mediator as well as a protective agent. The aim of this study was to evaluate the effect of IL- 6 deficiency on the expression of apoptotic regulatory proteins under both baseline conditions and following induction of ischemia and reperfusion in the mouse heart. C57BL/6J IL-6-/-TMKopf (IL6KO) and C57BL/6J mice (WT) were subjected to 30 minutes of local reversible myocardial ischemia in vivo or a sham operation. The expression of Bcl-2, Bax and STAT3 in the heart was assessed by western blotting. Under both baseline conditions and following the sham operation, IL-6 deficiency was associated with reduced expression of Bcl-2 and Bax. The TUNEL-FITC, Evans blue and tetrazolium chloride staining of the hearts following ischemia and reperfusion revealed similar injury in operated IL6KO and WT animals. There was increased STAT3 phosphorylation in operated mice regardless of the genotype. Bcl-2 and Bax expression was also comparable between the mouse strains following ischemia and reperfusion. In summary, these results indicated that IL-6 deficiency affected the basal expression of apoptotic regulators, but this did not profoundly alter the extent of reperfusion injury or apoptosis in the mouse heart following ischemia and reperfusion.
\end{abstract}

Key words:

heart, ischemia and reperfusion, interleukin 6, apoptosis, mice

\begin{abstract}
Abbreviations: CNTF - cilliary neurotrophic factor, DAPI 4',6-diamidino-2-phenylindole, IL-1 $\beta$ - interleukin 1 beta, IL-6 interleukin 6, IR - ischemia and reperfusion, JAK - Janus kinase, LIF - leukemia inhibitory factor, KO - knock-out, LCA - left coronary artery, LV - left ventricle, MAPK - mitogen activated protein kinase, PAGE - polyacrylamide gel electrophoresis, PBS - phosphate buffered saline, RIPA - radioimmunoprecipitation
\end{abstract}

assay, RNA - ribonucleic acid, SDS - sodium dodecyl sulfate, STAT - signal transducer activator of transcription, STEMI ST segment elevation myocardial infarction, TNF- $\alpha$ - tumor necrosis factor alpha, TTC - 2,3,5-triphenyltetrazolium chloride, TUNEL - terminal deoxynucleotidyltransferase-mediated dUTP nick end labeling, WT - wild type 


\section{Introduction}

Interleukin 6 (IL-6), cardiotrophin-1, leukemia inhibitory factor (LIF) and cilliary neurotrophic factor (CNTF) belong to a family of cytokines acting via the common receptor subunit gp130. IL-6 is a prototypic pleiotropic cytokine produced by various cell types, including leukocytes, fibroblasts, macrophages and endothelial cells. This cytokine is involved in mediating inflammation, antibody production, hormonal secretion, glucose metabolism, fatty acid turnover and many other physiological processes [23]. IL-6 is induced in the myocardium in response to harmful stimuli including ischemia [4], which is thought to contribute to the inflammatory reaction [16]. Interestingly, induction of IL- 6 expression is more rapid and more pronounced in the previously ischemic area where blood flow has been restored as compared to the non-reperfused tissue $[4,16]$.

Reperfusion therapy has become a gold standard treatment for ST-segment elevation myocardial infarction (STEMI) and has saved thousands of patients, but there is still room for improvement. Restoration of blood flow in the ischemic myocardium causes a series of events associated with rapid "oxidative burst", which is characterized by activation of the inflammatory process resulting in leukocyte infiltration and apoptosis [10]. Thus, reperfusion may, to some extent, aggravate the injury by increasing the rate of apoptosis of cardiac cells. Previous studies have suggested that the expression of pro-inflammatory cytokines, such as IL-6 and TNF $\alpha$, may be responsible for this activation of the inflammatory cascade and subsequent aggravation of the injury $[10,16]$. On the other hand, IL- 6 has been shown to inhibit the expression of pro-inflammatory cytokines (e.g., IL-1 $\beta$ ) and promote secretion of the anti-inflammatory cytokine IL10 [13]. Moreover, IL-6 exerts cytoprotective effects in other situations, such as hemorrhagic shock or ischemic preconditioning, by promoting the expression of the anti-apoptotic protein Bcl-2 [1, 21]. Gp130 is the major player that transduces IL-6-mediated signals from the extracellular environment to the intracellular environment. Gp130 is a membrane bound receptor that has no intrinsic kinase activity but has binding affinity for JAK kinases and proteins containing src-homology domains. Thus, JAK-STAT, Akt and MAPK signaling pathways are activated in response to IL-6 stimulation [23]. The first two pathways are vital for protecting myocardial tissue during reperfusion [6]. It has been reported that restoration of blood flow to the previously ischemic myocardium evokes a rapid increase in the rate of cardiac cell apoptosis [10]. It is widely accepted that this is the primary mechanism for persistent myocardial dysfunction following successful treatment of myocardial infarction [10]. The recent advances in understanding of the molecular mechanisms underlying ischemia and reperfusion injury have created excitement, since they have proposed that delivery of therapeutic agents against components of the apoptotic cascade can inhibit cell death and prevent injury [14]. IL-6 is one of the cytokines that is highly expressed in the myocardium following ischemia and reperfusion, so it may significantly influence the apoptosis of cells within the heart.

The aim of this study was to examine the effects of IL-6 deficiency on the regulation of apoptosis under baseline conditions and following myocardial injury in the murine model of regional cardiac ischemia and reperfusion.

\section{Materials and Methods}

Male C57BL/6J IL-6-/_TMKopf mice (IL6 KO) (body weight $27.8 \mathrm{~g} \pm 2.1 \mathrm{~g}$ ) and C57BL/6J (WT) control animals (body weight $27.9 \pm 2.1 \mathrm{~g}$ ) that were $12-16$ weeks old were used in this study.

Eight non-operated mice of each genotype were sacrificed by cervical dislocation, and their left ventricles were dissected to assess protein expression and apoptosis under baseline conditions.

A total number of 75 animals were subjected to sham operation $(\mathrm{SH})$ or to 30 minutes of regional reversible myocardial ischemia induced by ligation of the left anterior descending artery (IR group). The experimental model of ischemia and reperfusion was performed as previously described [6], with minor modifications. Briefly, mice were anesthetized with ketamine and xylazine ip $(120 \mathrm{mg} / \mathrm{kg}$ and $3 \mathrm{mg} / \mathrm{kg}$, respectively), placed on a heating pad at a constant temperature of $37^{\circ} \mathrm{C}$, intubated and ventilated with a mouse respirator (Minivent, Harvard Instruments) using oxygen-enriched air (volume of $0.25 \mathrm{ml}$ and frequency of $200 / \mathrm{min}$ ). A left-sided lateral thoracotomy in the 5 th intercostal space was performed, which was followed by exposure of the anterior wall of the left 
ventricle (LV). A prolene 6-0 suture was placed around the left coronary artery (LCA), and it was tied to polyethylene tubing causing occlusion of the LCA. After 30 minutes, the knot was cut and the tubing was removed, so that the blood flow was restored. Reperfusion was assessed by visually monitoring whether there was blushing of the previously pale myocardium. The chest was closed in layers, and the muscles were repositioned. The mice were ventilated until spontaneous breathing commenced. They were transferred to a cage with a temperature of $27^{\circ} \mathrm{C}$ for recovery and were provided with water and chow ad libitum. The sham operation $(\mathrm{SH})$ was precisely performed as described above, with the exception of tying the suture. Thus, the length of the $\mathrm{SH}$ procedure was identical to the IR group, except that the LV was not subjected to ischemia and reperfusion. Eight mice died during the perioperative period so 67 animals were used for further analysis.

For the assessment of apoptosis, 24 mice were sacrificed by cervical dislocation 5 hours after reperfusion (or sham operation). The hearts were dissected, and the apex of the left ventricle (LV) was embedded in Jung tissue freezing medium (OCT) and frozen in liquid nitrogen. The basis of the LV was fixed in formalin and embedded in paraffin. At the same time point, an additional 6 mice from each of the 4 groups (two genotypes, sham and IR) were sacrificed by cervical dislocation for the analysis of protein expression in the left ventricle. Myocardial samples were taken from the apical region of left ventricles of nonoperated and sham-operated animals, as well as from the ischemic area and remote LV myocardium of the IR group. We have chosen a 5 hour time point based on a study by Dawn et al. [3], who reported that there is very high expression of IL-6 2 hours after ischemia and reperfusion. Based on this observation, we expected early IL-6 dependent changes to occur 3 hours after the increase in IL-6 expression. The specimens were snap frozen in liquid nitrogen and stored in $-70^{\circ} \mathrm{C}$ until further analysis.

A total of 9 WT and 10 IL6 KO mice were sacrificed 24 hours after reperfusion for assessment of infarct size by Evans blue and 2,3,5-triphenyltetrazolium chloride (TTC) staining as previously described [5]. Briefly, mice were anesthetized again, then intubated and connected to the respirator. The LCA was permanently occluded in the exact location of the previous ligation. The aorta was canulated, and the heart was removed. Evans blue (Sigma) (1 $\mathrm{ml}$ of $1 \%$ solution in phosphate buffered saline) was infused into the coronary system via the aorta to delineate the ischemic zone. The LV was dissected, cut into 6-8 transverse slices, and incubated for 15 minutes in $2 \%$ TTC at $37^{\circ} \mathrm{C}$. Thus, the non-ischemic area stained blue, viable myocardium stained brick-red, and the infarct region remained pale. Subsequently, slices were fixed in phosphate buffered $3.4 \%$ formalin for 24 hours. They were weighed and photographed from both sides using a digital camera. The Image Tool 3.0 (UTHSCSA USA) software was used to evaluate the areas of nonischemic myocardium, area at risk and infarct area. The proportional weights of infarct and area at risk of whole hearts were calculated using the weights of the slices and proportions of areas at risk, infarction and non-ischemic left ventricle of each respective slice. A ratio of infarct to area at risk was used as a parameter to represent the extent of ischemia and reperfusion injury. In two cases (one from each group), the staining failed.

The OCT embedded frozen tissue was cut transversally and subjected to terminal transferase dUTP nick end labeling (TUNEL) with fluorescein using the ApopTag kit, according to the manufacturer's protocol (Chemicon) [12]. All slides were counterstained with DAPI (Chemicon) for the assessment of nuclear morphology. Images of the ischemic and reperfused areas of the left ventricle on each slide (approximately 5 fields per slide) were acquired using a fluoroscopic microscope (Olympus). The ratio of the number of FITC stained nuclei (with the confirmed apoptotic morphology from DAPI staining) and all DAPIstained nuclei represented a measure of apoptosis. The same procedure was performed on formalin fixed paraffin embedded tissue. All assessments were performed by a person blinded to the group assignment of animals.

Western blotting was performed as previously described [12]. Myocardial samples from the left ventricle were snap-frozen in liquid nitrogen. They were later homogenized on ice in RIPA buffer (Sigma) containing protease and phosphatase inhibitors (Sigma) using a mechanical disperser (Ika T10). The homogenates were centrifuged at $4^{\circ} \mathrm{C}$ for 20 minutes at $12,000 \times \mathrm{g}$. The supernatant was collected, and the protein content was measured using Bradford reagent (Sigma). Samples were frozen at $-70^{\circ} \mathrm{C}$ until further analysis. Protein extracts were subjected to SDSPAGE and transferred to nitrocellulose membranes (BioRad) for western blotting. Equal loading was 
confirmed using Ponceau Red staining (Sigma). Duplicate membranes were probed with primary antibodies recognizing mouse STAT3 (Santa Cruz, 1:500), phosphorylated STAT3 (Santa Cruz, 1:500), Bcl-2 and Bax (Sigma, both 1:1000). Secondary antibodies conjugated to horseradish peroxidase (Serotec) were used. Blots were visualized using enhanced chemiluminescence (ImmunStar, BioRad) and exposed to x-ray film (X-Omat, Blue Kodak). Results were scanned and quantified using Scion Image software (Scion Corporation). Protein abundance in all animals was quantified in relation to a single WT sham-operated animal, in which expression of the particular protein was arbitrarily set as 1 . Therefore, both groups had variation, which is represented as standard deviation (SD).

The experimental procedures were performed on the extensively studied C57BL/6J IL-6-/_TMKopf strain $[5,11,19,22]$, and results were compared to the respective wild type $\mathrm{C} 57 \mathrm{BL} / 6 \mathrm{~J}$ animals. The genotypes of the founders and randomly chosen experimental mice were assessed as previously described using polymerase chain reaction based analysis of DNA isolated from mouse tails [19]. All the animals used in the study had the correct genotype.

The experimental procedures were carried out according to the European Council Directive of 24 November 1986 (6/609/EEC) and were approved by the Local Ethics Committee in Białystok.

\section{Statistical analysis}

Results were presented as the mean \pm standard deviation (SD). The Student $t$-test was used to evaluate statistical analysis. A p value less than 0.05 was considered statistically significant.

\section{Results}

The expression of the anti-apoptotic protein Bcl-2 in the non-operated animals was significantly lower in the IL6 KO animals as compared to their respective WT mice (Fig. 1A) $(0.49 \pm 0.31 v s .1 \pm 0.24$, respectively, $\mathrm{p}<0.01)$. Similarly, the expression of the proapoptotic protein Bax was significantly lower in the IL6 KO animals as compared to their respective WT mice (Fig. 1B) $(0.65 \pm 0.2$ vs. $1 \pm 0.09$, respectively, $\mathrm{p}<0.01)$.
The myocardial samples acquired from nonoperated or sham-operated animals presented only single TUNEL-positive nuclei per cross section regardless of the genotype, so they were not quantified (data not shown). In contrast, there were multiple TUNEL positive nuclei in the samples obtained from the animals subjected to ischemia and reperfusion (IR group). The assessment of apoptosis by TUNELFITC staining on OCT-embedded frozen tissue showed a similar percentage of apoptotic nuclei in the area at risk for both the IL6 KO and WT animals (IR group) (Fig. 2). Assessment of apoptosis by TUNEL staining of formalin-fixed paraffin-embedded tissue confirmed that the frequency of apoptosis was comparable between both groups (data not shown).

Evans Blue and TTC staining were performed 24 hours after 30 minutes of regional ischemia by LCA ligation followed by reperfusion. The analysis revealed a comparable area at risk of the LV in both IL6 KO and WT animals. Moreover, the infarct to LV mass ratio was almost identical. Hence, there were no significant differences in the parameter widely accepted as the extent of ischemia-reperfusion injury: the ratio of infarct to area at risk (Fig. 3).

The expression of the anti-apoptotic protein $\mathrm{Bcl}-2$ in the sham-operated animals and in the remote myocardium of the IR mice was significantly lower in the IL6 KO animals as compared to their respective WT mice (Fig. 4A) (in Sham $0.4 \pm 0.2$ vs. $1.2 \pm 0.3$, respectively, and in remote myocardium $0.6 \pm 0.2 \mathrm{vs}$. $1.1 \pm 0.5$, respectively). However, in the area of the left ventricle subjected to ischemia and reperfusion, the expression of this protein was significantly increased as compared to the sham-operated animals for both WT and IL6 KO mice, and the differences between the genotypes were no longer significant (Bcl-2 relative expression: $1.9 \pm 0.9$ vs. $1.3 \pm 0.2$, respectively) (Fig. 4A). For both genotypes, the expression of $\mathrm{Bcl}-2$ in the ischemic and reperfused areas was significantly higher than in the remote myocardium $(p<0.05)$.

Interestingly, the expression of the pro-apoptotic protein Bax in the sham-operated animals was also significantly lower in the IL6 KO animals as compared to their respective WT mice (Fig. 4B) (in Sham $0.27 \pm 0.1$ vs. $1.35 \pm 0.39$, respectively, $\mathrm{p}<0.01$ ). The expression of Bax was significantly increased in the ischemic and reperfused part of the left ventricle as compared to sham-operated WT and IL6 KO mice $(p<0.05)$. Nevertheless, the genotypic difference in 


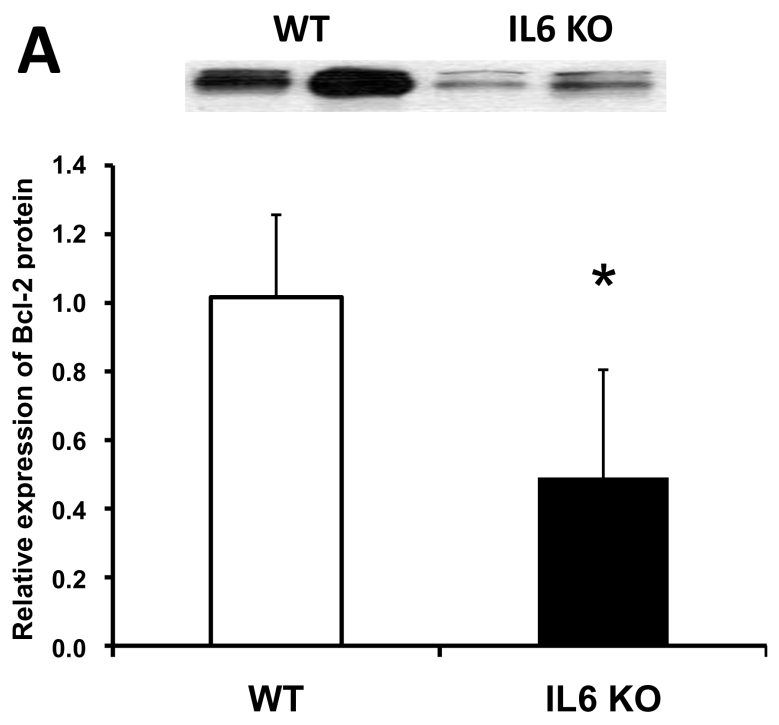

B
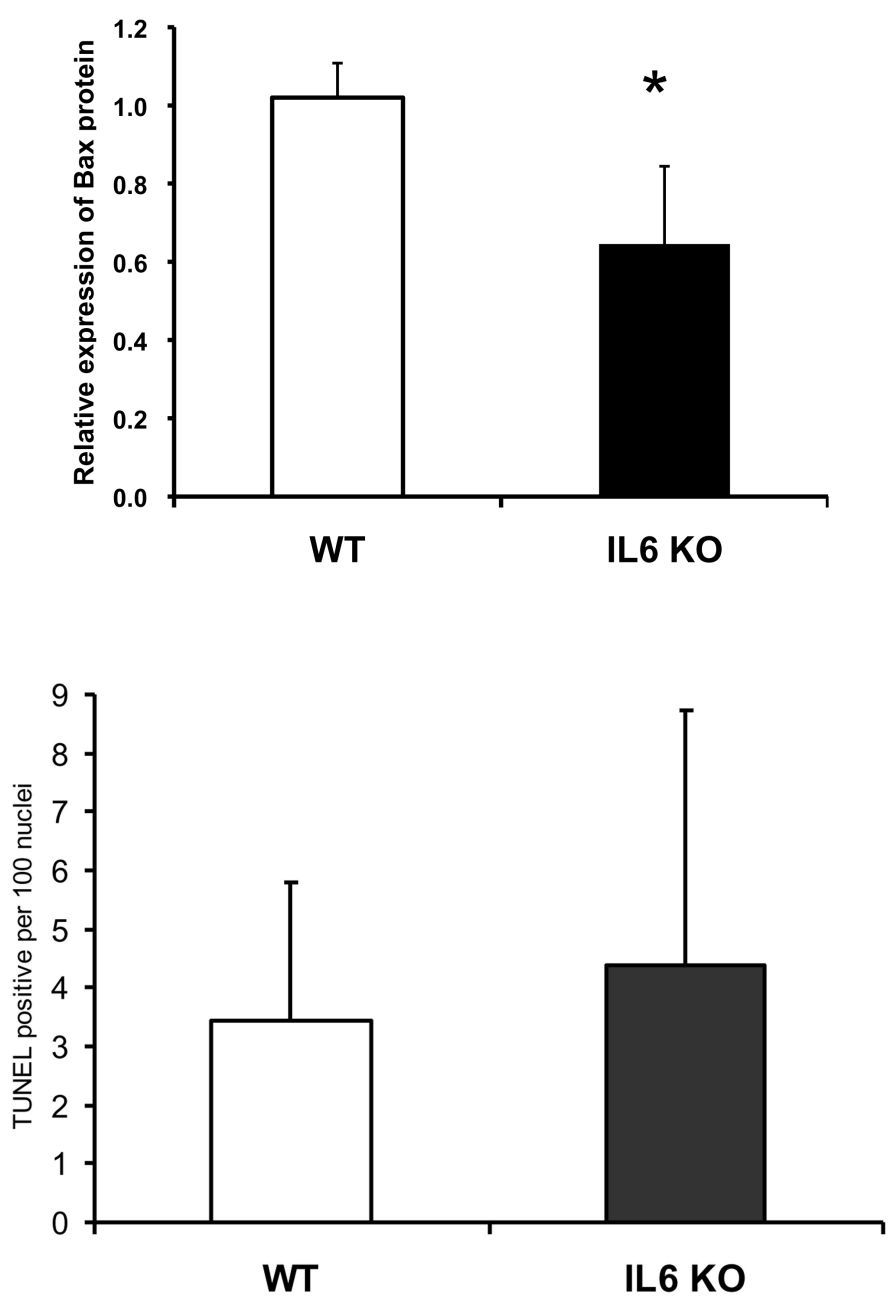

Fig. 1. Western blots and densitometric analysis presenting relative protein expression of $\mathrm{Bcl}-2(\mathbf{A})$ and $\mathrm{Bax}(\mathbf{B})$ in the left ventricles of non-operated C57BL6 $(\mathrm{WT} n=8)$ and IL-6 knock-out (IL6 KO $\mathrm{n}=8)$ mice. Columns represent the mean \pm standard deviation. ${ }^{*} p<0.05$ vs. respective WT group

Fig. 2. Apoptosis in the area at risk of the heart after 30 minutes of regional ischemia and 5 hours of reperfusion in C57BL6 (WT $n=8$ ) and IL-6 knock-out (IL6 $n=8$ ) mice, as assessed by TUNEL-FITC staining, represented as the ratio between TUNEL-positive nuclei and DAPI-stained nuclei. Columns represent the mean \pm standard deviation 


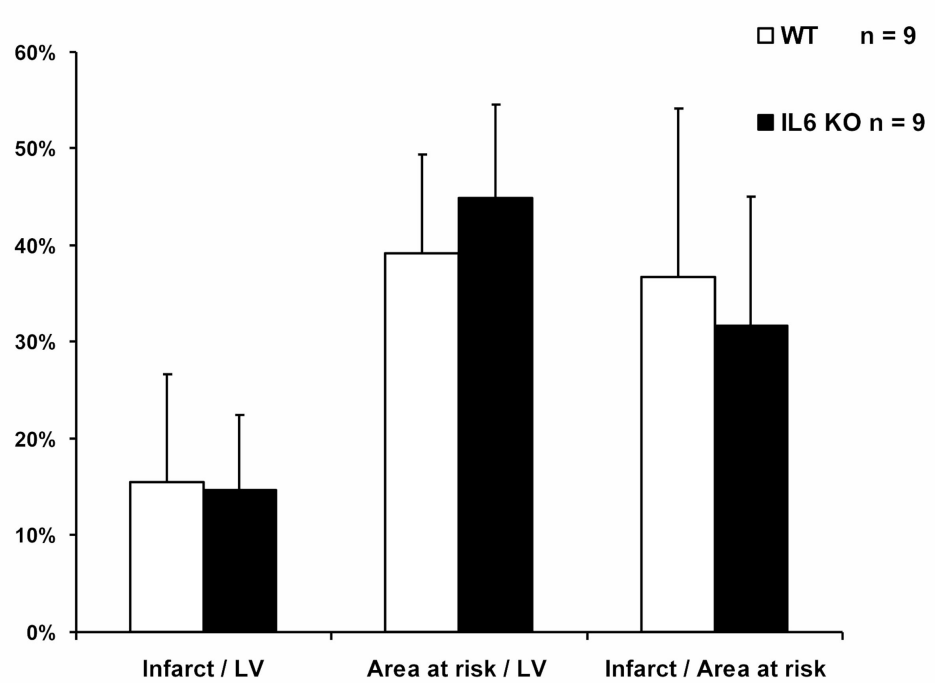

A $\frac{\text { Sham }}{\text { IL6 KO WT }} \frac{\text { Remote myocardium }}{\text { IL6 KO Ischemic myocardium }}$
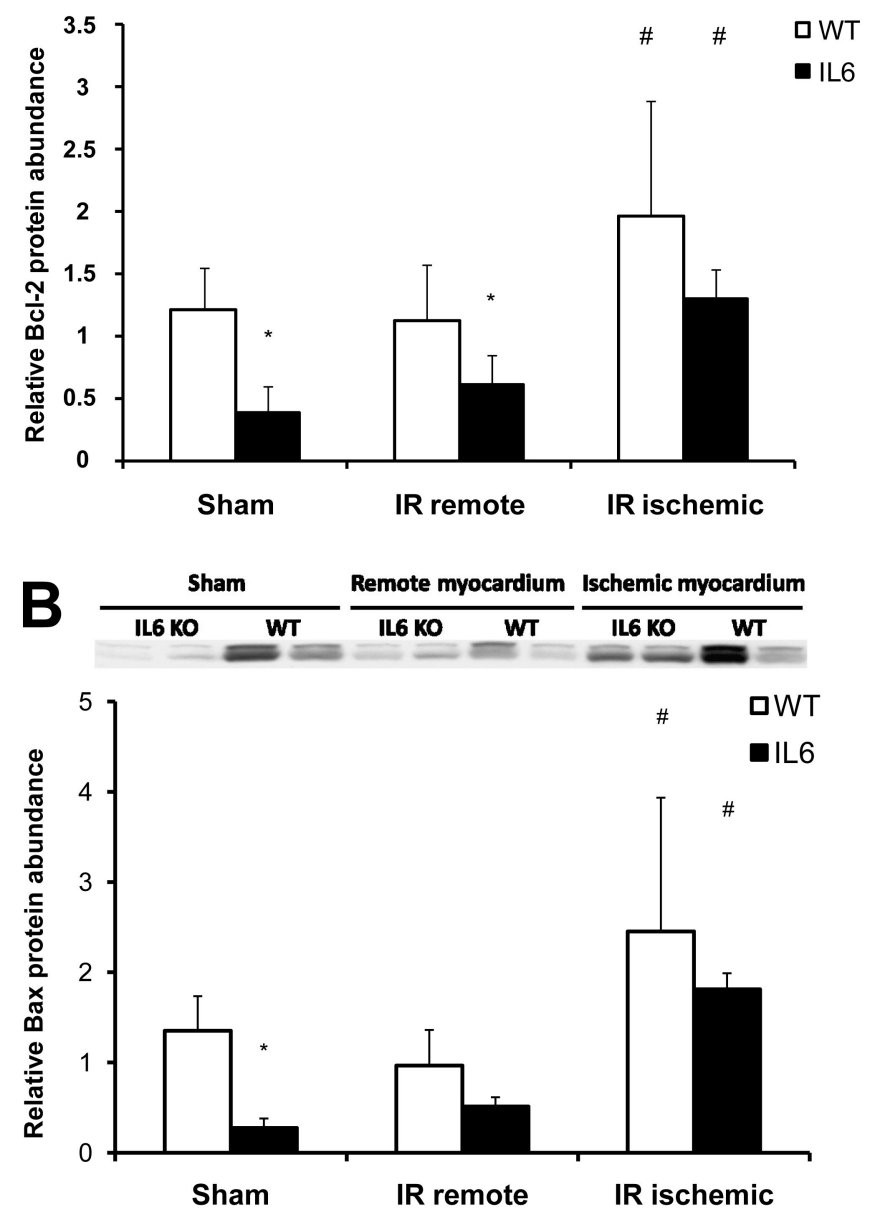

Fig. 3. Relation of infarct and area at risk masses to the left ventricle mass and infarct to area at risk ratio in C57BL6 (WT $n=8)$ and IL-6 knock-out (IL6 KO $n=9$ ) mice 24 hours after ischemia and reperfusion, as measured by Evans Blue and TTC staining. Columns represent the mean \pm standard deviation

Fig. 4. Western blots and densitometric analysis presenting the relative protein expression of $\mathrm{BCl}-2(\mathbf{A})$ and $\mathrm{Bax}(\mathbf{B})$ in the left ventricles of sham-operated animals (Sham $n=6$ for each genotype) in the non-ischemic myocardium (IR remote) and ischemic area of the left ventricle (IR ischemic) of C57BL6 $(\mathrm{WT} n=6)$ and IL-6 knock-out (IL6 KO n = 6) mice 5 hours after ischemia and reperfusion. Columns represent the mean \pm standard deviation. * $p<0.05$ vs. respective WT group; $\# p<0.05$ vs. IR remote myocardium in the respective groups 
Bax expression in this area was no longer significant $(2.4 \pm 1.5$ in WT vs. $1.8 \pm 0.18$ in IL6 KO) (Fig. 4B).

The expression of total STAT3 protein was comparable in sham-operated WT and IL6 KO animals and was not affected by ischemia and reperfusion for either genotype (Fig. 5A). The phosphorylation of STAT3 protein was significantly increased in the ischemic and reperfused area as compared to sham-operated animals or the remote myocardium of animals subjected to ischemia-reperfusion injury (Fig. 5B) ( $p<0.05$ ). However, there were no significant differences between genotypes in respective samples (Fig. 5B) (relative STAT3-P expression: $3.6 \pm 0.9$ in WT animals after ischemia and reperfusion, and $4.3 \pm 1.5$ in IL6 KO after ischemia and reperfusion).

\section{Discussion}

The results of this study suggest that IL-6 deficiency is an important factor that affects the expression of pro and anti-apoptotic factors under physiological conditions. Nevertheless, it has no significant influence on the regulation of myocardial apoptosis in mice and does not affect tissue injury resulting from ischemia and reperfusion. Similar results have been previously published by Dawn et al. [3]; they described a comparable infarct to area at risk ratio in IL6 KO mice as compared to WT animals. However, they did not assess the extent of apoptosis or the expression of regulatory proteins. Compatible results, using

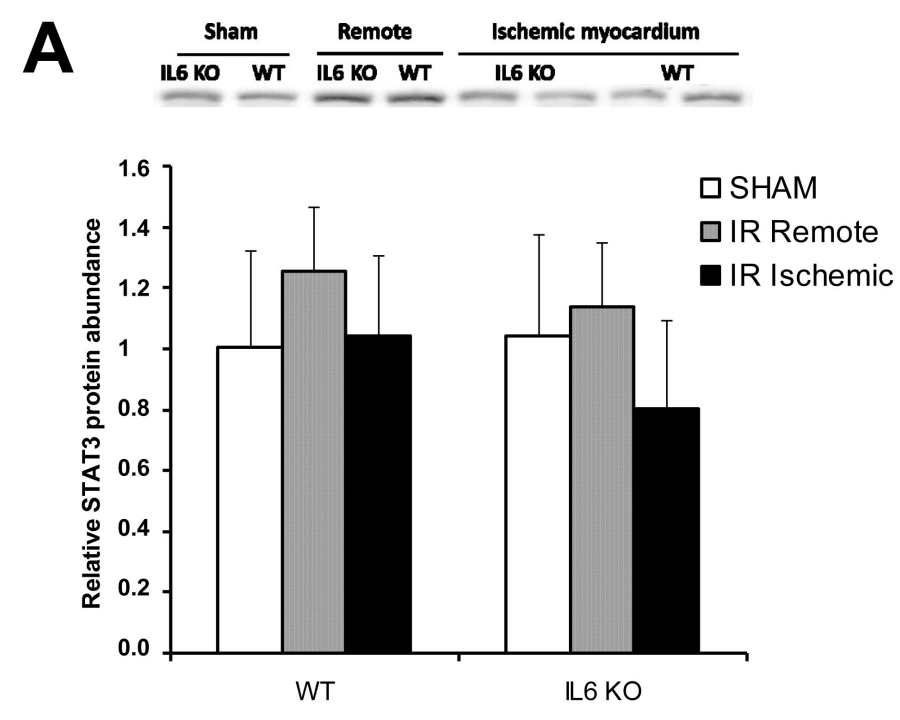

B

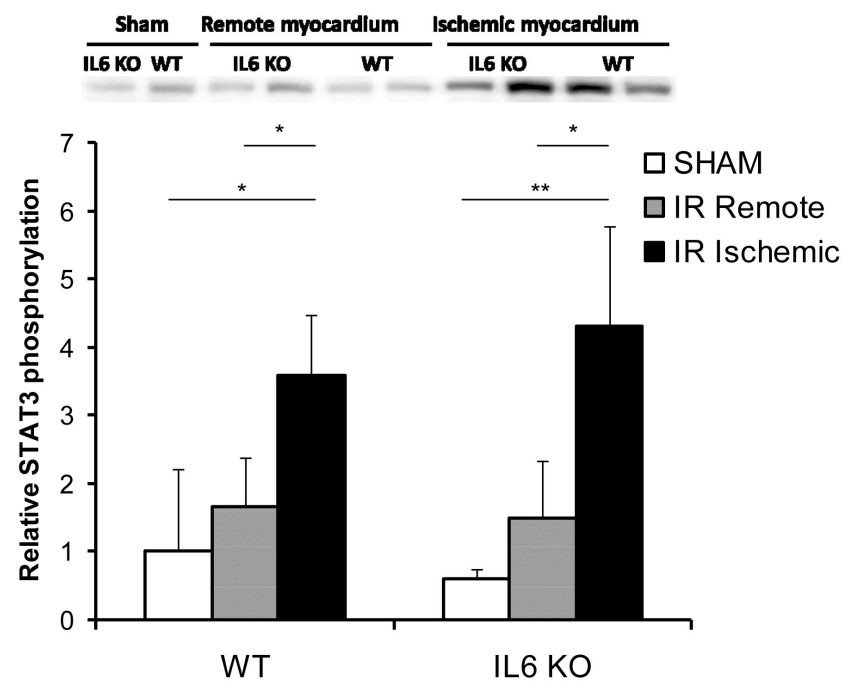

Fig. 5. Western blots and densitometric analysis presenting expression of total STAT3 protein $(\mathbf{A})$ and phosphorylated STAT3 (B) in the ischemic area of the left ventricle (IR ischemic) and in the remote myocardium (IR remote) of C57BL6 (WT) and IL-6 knock-out (IL6) mice 5 hours after ischemia and reperfusion $(n=6$ for each genotype) or after a sham operation (SHAM $n=6$ for each genotype). Columns represent the mean \pm standard deviation. ${ }^{*} p<0.05$ Student $t$-test ; ${ }^{* *} p<0.01$ Student $t$-test 
a different model, have been presented by Fuchs et al. [5]. They have shown that IL-6 deficiency had no effect on infarct size and LV remodeling following permanent LCA ligation in mice. Similarly to our findings, they have noticed similar phosphorylation levels of STAT3 after infarction in both IL6 KO and WT animals. In our study, this comparable activation of STAT3 correlated with similar expression of the antiapoptotic protein $\mathrm{Bcl}-2$ in the ischemic and reperfused myocardium. This finding is consistent with our TUNEL-FITC staining results in the area at risk, which demonstrated a similar extent of apoptosis in both IL6 KO and WT animals. Moreover, we did not observe any difference in the expression level of the pro-apoptotic protein Bax between genotypes. This finding also supports that during severe injury, other signaling factors assume regulation of apoptosis.

Interestingly, a very different expression pattern was observed in the non-operated controls as well as in the sham-operated animals and in areas of the myocardium that were not subjected to hypoxia. In those regions, the expression of $\mathrm{Bcl}-2$ was significantly lower in the IL6 KO animals as compared to WT mice. The expression of Bax was significantly reduced in non-operated and sham-operated IL6 KO animals as compared to their WT counterparts, whereas Bax expression in the remote myocardium of the operated animals was comparable for both genotypes. Those results indicated that IL- 6 is not vital for increased Bcl-2 and Bax expression after ischemia and reperfusion in mouse cardiac tissue. On the other hand, baseline conditions or mild injury (e.g., sham operation) are associated with lower Bcl-2 expression in the absence of IL-6, which suggests that this cytokine is involved in the up-regulation of expression of antiapoptotic proteins in the context of mild generalized injury. Similar results have been described in enterocytes during ischemia [21], in pulmonary epithelium after hyperoxia [25] and in hepatocytes after alcohol exposition [7] or Fas treatment [15]. The Bcl-2 expression in cells exposed to relatively mild stressful stimuli (sham operation, which was used in our study, alcohol consumption, hyperoxia or simulated ischemia) was significantly lower in the absence of IL-6. Bax expression seems to depend to some extent on the presence of IL-6 only under physiological conditions.

Interestingly, after more severe challenges (cardiac ischemia and reperfusion), there were no differences, probably because of increased expression of other proteins that affect Bcl-2 and Bax expression. One may attribute this effect to putative compensatory mechanisms in animals devoid of functional IL-6 gene expression during their lifetime. Moreover, we found no difference in STAT3 phosphorylation, which suggests that there are increased levels of substances activating this signal transduction pathway. It has been previously described that myocardial infarction in IL-6 KO mice caused increased expression of other IL-6 family members, including LIF (leukemia inhibitory factor) [5]. This cytokine has been shown to induce STAT3 phosphorylation and prevent apoptosis by inducing overexpression of Bcl family members [20]. Simultaneously, various pro-apoptotic substances are induced that may up-regulate Bax expression in an IL-6-independent manner during ischemia and reperfusion [4].

There is support for the theory that IL-6 is the first-line signaling cytokine in the heart induced in response to relatively mild stressful stimuli. Dawn et al. [3] have shown that IL-6 is highly expressed after very short periods of ischemia, and it conveys signals that are crucial for cytoprotection. IL-6 deficiency in mice resulted in lack of ischemic preconditioning. Given these observations, our results confirmed the hypothesis that IL-6 signaling is overridden in the case of prolonged ischemia and deleterious reperfusion.

The role of IL-6 in the cardiovascular system is very complex and is far from being fully elucidated. On one hand, it has been previously shown that an increased serum level of IL-6 is associated with a higher probability of MI and subsequent cardiovascular death [18], and development of congestive heart failure [24]. On the other hand, IL-6 has been shown to mediate some of the anti-inflammatory and metabolic effects of exercise both in clinical and experimental studies [23]. Hence, it is still not fully understood whether association of IL-6 with the poor prognosis of cardiovascular patients is an accompanying phenomenon, a compensatory mechanism, or if it exerts profound detrimental effects.

Interestingly, the observed phenomena may provide insight on the effect of increased IL-6 levels on distant organs such as the brain. The recently described behavioral changes in IL-6 KO mice [8] may, to some extent, resemble phenomena observed in mice with lower expression of Bcl-2 in their brain [17].

The IL-6 transduction cascade may be considered a very precise, finely-tuned signaling system. Interestingly, IL-6 deficiency often results in similar effects to those induced by IL-6 overexpression [9, 22], 
which makes a J-shape or U-shape dose response curve effect plausible. Moreover, the rapid and shortlasting release of IL-6 may have different metabolic effects than long lasting release, as demonstrated by Carey et al. [2]. IL-6 secreted by contracting muscle is considered to diminish insulin resistance and improve glucose metabolism, whereas long-lasting IL-6 elevation is clearly detrimental and closely associated with impairment of insulin signaling [2]. Interestingly, despite the large body of evidence on the effects of exercise-induced IL-6 secretion on insulin-mediated signaling and potentially on physiologic hypertrophy, there is no influence of IL-6 deficiency on exerciseinduced cardiac hypertrophy in mice [11].

Apart from the new insights into the pathogenesis of ischemia-reperfusion injury, this study has a limitation as we were not able to identify the factors which replace the function of IL- 6 on induction of $\mathrm{Bcl}-2$ expression in the context of cardiac ischemia and reperfusion. Nevertheless, in our opinion, our results provided valuable insight on the role of IL-6 in the regulation of apoptosis in cardiac tissue and in the pathogenesis of reperfusion injury. However, further studies are necessary to clarify the role of IL-6 in the heart.

\section{Conclusions}

IL6 deficiency significantly affects the expression of proteins regulating apoptosis under physiological conditions or mild stress, such as sham operation. However, IL-6 deficiency does not affect apoptosis or the extent of reperfusion injury following cardiac ischemia and reperfusion in mice.

\section{Acknowledgments}

The authors are exceptionally grateful to Professors Denise Hilfiker-Kleiner and Helmut Drexler for their generous gift of the C57BL6/J IL-6-/- ${ }^{\text {TMKopf }}$ mouse strain. The authors thank Mrs. Agnieszka Leszczyńska for her excellent technical assistance. This project was supported by a grant from The Polish Ministry of Science (2P05B 01826) and a grant from Medical University of Bialystok (no. 3-24598).

\section{References:}

1. Arikan AA, Yu B, Mastrangelo MA, Tweardy DJ: Interleukin-6 treatment reverses apoptosis and blunts sus- ceptibility to intraperitoneal bacterial challenge following hemorrhagic shock. Crit Care Med, 2006, 34, 771-777.

2. Carey AL, Steinberg GR, Macaulay SL, Thomas WG, Holmes AG, Ramm G, Prelovsek O et al.: Interleukin-6 increases insulin-stimulated glucose disposal in humans and glucose uptake and fatty acid oxidation in vitro via AMPactivated protein kinase. Diabetes, 2006, 55, 2688-2697.

3. Dawn B, Xuan YT, Guo Y, Rezazadeh A, Stein AB, Hunt $\mathrm{G}, \mathrm{Wu}$ WJ et al.: IL-6 plays an obligatory role in late preconditioning via JAK-STAT signaling and upregulation of iNOS and COX-2. Cardiovasc Res, 2004, 64, 61-71.

4. Deten A, Volz HC, Briest W, Zimmer HG: Cardiac cytokine expression is upregulated in the acute phase after myocardial infarction. Experimental studies in rats. Cardiovasc Res, 2002, 55, 329-340.

5. Fuchs M, Hilfiker A, Kaminski K, Hilfiker-Kleiner D, Guener Z, Klein G, Podewski E et al.: Role of interleukin-6 for LV remodeling and survival after experimental myocardial infarction. FASEB J, 2003, 17, 2118-2120.

6. Hilfiker-Kleiner D, Hilfiker A, Fuchs M, Kaminski K, Schaefer A, Schieffer B, Hillmer A et al.: Signal transducer and activator of transcription 3 is required for myocardial capillary growth, control of interstitial matrix deposition, and heart protection from ischemic injury. Circ Res, 2004, 95, 187-195.

7. Hong F, Kim WH, Tian Z, Jaruga B, Ishac E, Shen X, Gao B: Elevated interleukin-6 during ethanol consumption acts as a potential endogenous protective cytokine against ethanol-induced apoptosis in the liver: involvement of induction of $\mathrm{Bcl}-2$ and $\mathrm{Bcl}-\mathrm{x}(\mathrm{L})$ proteins. Oncogene, 2002, 21, 32-43.

8. Hryniewicz A, Bialuk I, Kaminski KA, Winnicka MM: Impairment of recognition memory in interleukin-6 knock-out mice. Eur J Pharmacol, 2007, 577, 219-220.

9. Huber SA, Sakkinen P, Conze D, Hardin N, Tracy R: Interleukin-6 exacerbates early atherosclerosis in mice. Arterioscler Thromb Vasc Biol, 1999, 19, 2364-2367.

10. Kaminski KA, Bonda TA, Korecki J, Musial WJ: Oxidative stress and neutrophil activation - the two keystones of ischemia/reperfusion injury. Int J Cardiol, 2002, 86, 41-59.

11. Kaminski KA, Olędzka E, Białobrzewska K, Kożuch M, Musiał WJ, Winnicka MM: The effects of moderate physical exercise on cardiac hypertrophy in interleukin 6 deficient mice. Adv Med Sci, 2007, 52, 164-168.

12. Kamiński KA, Usowicz-Szaryńska M, Kożuch M, Bonda T, Karwowski W, Chyczewski L, Sawicki B et al.: Preconditioning with the low dose of lipopolysaccharide attenuates apoptosis in heart during septic shock in mice. Pharmacol Rep, 2007, 59 (Suppl 1), 161-166.

13. Kopf M, Baumann H, Freer G, Freudenberg M, Lamers M, Kishimoto T, Zinkernagel R et al.: Impaired immune and acute-phase responses in interleukin-6-deficient mice. Nature, 1994, 368, 339-342.

14. Kovacs P, Bak I, Szendrei L, Vecsernyes M, Varga E, Blasig IE, Tosaki A: Non-specific caspase inhibition reduces infarct size and improves post-ischaemic recovery in isolated ischaemic/reperfused rat hearts. Naunyn Schmiedebergs Arch Pharmacol, 2001, 364, 501-507. 
15. Kovalovich K, Li W, DeAngelis R, Greenbaum LE, Ciliberto G, Taub R: Interleukin-6 protects against Fasmediated death by establishing a critical level of antiapoptotic hepatic proteins FLIP, Bcl-2, and Bcl-xL. J Biol Chem, 2001, 276, 26605-26613.

16. Kukielka GL, Smith CW, Manning AM, Youker KA, Michael LH, Entman ML: Induction of interleukin-6 synthesis in the myocardium. Potential role in postreperfusion inflammatory injury. Circulation, 1995, 92, 1866-1875.

17. Lien R, Flaisher-Grinberg S, Cleary C, Hejny M, Einat $\mathrm{H}$ : Behavioral effects of Bcl-2 deficiency: implications for affective disorders. Pharmacol Rep, 2008, 60, 490-498.

18. Luc G, Bard JM, Juhan-Vague I, Ferrieres J, Evans A, Amouyel P, Arveiler D et al.: C-reactive protein, interleukin-6, and fibrinogen as predictors of coronary heart disease: the PRIME Study. Arterioscler Thromb Vasc Biol, 2003, 23, 1255-1261.

19. Mysliwiec J, Zbucki R, Winnicka M, Sawicki B, Nikolajuk A, Kaminski K, Mysliwiec P et al.: Interleukin-6 is not essential for bone turnover in hypothyroid mice. Folia Histochem Cytobiol, 2007, 45, 387-392.

20. Negoro S, Kunisada K, Tone E, Funamoto M, Oh H, Kishimoto T, Yamauchi-Takihara K: Activation of JAK/STAT pathway transduces cytoprotective signal in rat acute myocardial infarction. Cardiovasc Res, 2000, 47, 797-805.
21. Rollwagen FM, Madhavan S, Singh A, Li YY, Wolcott $\mathrm{K}$, Maheshwari R: IL-6 protects enterocytes from hypoxia-induced apoptosis by induction of bcl-2 mRNA and reduction of fas mRNA. Biochem Biophys Res Commun, 2006, 347, 1094-1098.

22. Schieffer B, Selle T, Hilfiker A, Hilfiker-Kleiner D, Grote K, Tietge UJ, Trautwein C et al.: Impact of interleukin- 6 on plaque development and morphology in experimental atherosclerosis. Circulation, 2004, 110, 3493-3500.

23. Song M, Kellum JA: Interleukin-6. Crit Care Med, 2005, 33, S463-465.

24. Vasan RS, Sullivan LM, Roubenoff R, Dinarello CA, Harris T, Benjamin EJ, Sawyer DB et al.: Inflammatory markers and risk of heart failure in elderly subjects without prior myocardial infarction: the Framingham Heart Study. Circulation, 2003, 107, 1486-1491.

25. Ward NS, Waxman AB, Homer RJ, Mantell LL, Einarsson $\mathrm{O}$, Du Y, Elias JA: Interleukin-6-induced protection in hyperoxic acute lung injury. Am J Respir Cell Mol Biol, 2000, 22, 535-542.

Received:

November 30, 2008; in revised form: May 11, 2009 